\title{
Development of automated angle-scanning, high-speed surface plasmon resonance imaging and SPRi visualization for the study of dropwise condensation
}

\author{
Shahab Bayani Ahangar ${ }^{1} \cdot$ Vinaykumar Konduru $^{1}$. Jeffrey S. Allen ${ }^{1} \cdot$ Nenad Miljkovic $^{2} \cdot$ Seong Hyuk Lee $^{3}$. \\ Chang Kyoung Choi ${ }^{1}$
}

Received: 30 May 2019 / Revised: 31 October 2019 / Accepted: 5 November 2019 / Published online: 3 December 2019

(c) The Author(s) 2019

\begin{abstract}
This paper describes the fabrication and testing of a novel angle-scanning surface plasmon resonance imaging (SPRi) instrument. The combination of two stationary mirrors and two angle-controlled mirrors provides high accuracy (up to $10^{-30}$ ) and high-speed angular probing. This instrument minimizes the angle-dependent image artifact that arises due to beam walk, which is the biggest challenge for the use of SPRi with angular modulation (AM). In the work described in this paper, two linear stages were employed to minimize the image artifact by adjusting the location of the angle-controlled mirrors and the camera. The SPRi instrument was used to visualize coalescence during dropwise condensation. The results show that the effect of the environment's temperature on reflectance was less than $1 \%$ when the incident angle was carefully chosen for SPRi with intensity modulation (IM). This means that condensation visualization can be carried out at ambient temperatures, without the need for a Peltier stage or a thermally controlled condensing surface. The concept of pixel neighboring was employed to assess the probability of noise and the standard error of thin film measurement. Experimental analyses during dropwise condensation show (1) the presence of a thin film with thickness of one monolayer, and (2) surface coverage of $0.71 \mathrm{~m}^{2} / \mathrm{m}^{2}$ by the thin film in the area between the droplets. In addition, analyses showed the existence of a dry area at the part of the substrate exposed by coalescence to ambient air. The results of this work undermine the validity of the film rupture theory as the dropwise condensation mechanism.
\end{abstract}

\section{Graphic abstract}

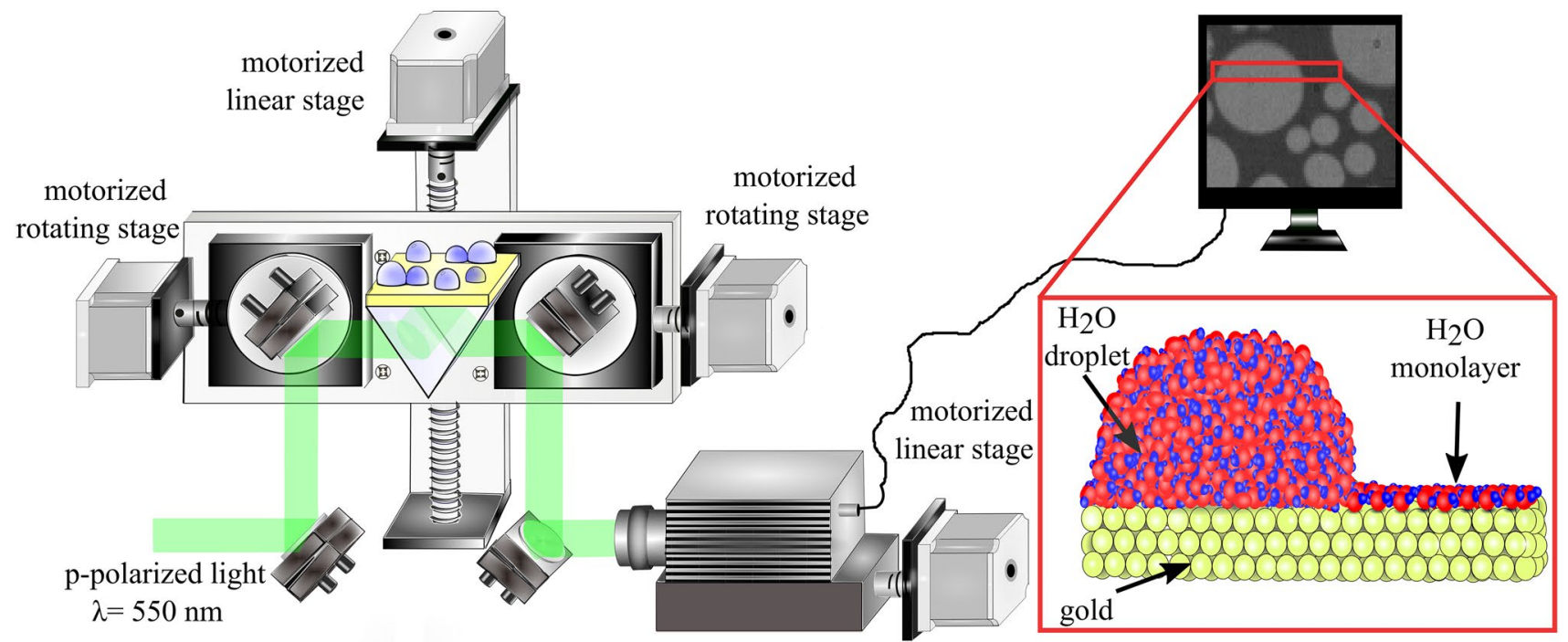

Extended author information available on the last page of the article 


\section{List of symbols}

$d \quad$ Thickness (nm)

$k_{0} \quad$ Proportionally constant (molecules $/ \mathrm{cm}^{2} \mathrm{~s}$ )

$k_{\mathrm{a}} \quad$ Adsorption coefficient

$k_{\text {al }} \quad$ Adsorption coefficient at the liquid-vapor interface

$k_{\text {as }} \quad$ Adsorption coefficient at the solid-vapor interface

$K_{\mathrm{B}} \quad$ Boltzmann coefficient [1.38E-23(J/K)]

$n \quad$ Refractive index

$P \quad$ Pressure $(\mathrm{Pa})$

$R \quad$ Gas constant $[8.314(\mathrm{~J} / \mathrm{mol} \mathrm{K})]$

$r_{\mathrm{a}} \quad$ Rate of adsorption (molecules $/ \mathrm{cm}^{2} \mathrm{~s}$ )

$r_{\mathrm{d}} \quad$ Rate of desorption (molecules $/ \mathrm{cm}^{2} \mathrm{~s}$ )

$R_{\mathrm{SPR}} \quad$ SPR reflectance

$T \quad$ Temperature (K)

$w \quad$ Energy per mole required to evaporate adsorbed molecules $(\mathrm{J} / \mathrm{mole})$

$w_{\mathrm{s}} \quad$ Energy per mole required to evaporate adsorbed molecules at the solid-vapor interface $(\mathrm{J} / \mathrm{mole})$

$w_{1} \quad$ Energy per mole required to evaporate adsorbed molecules at the liquid-vapor interface $(\mathrm{J} / \mathrm{mole})$

$\alpha \quad$ Incident angle $\left(^{\circ}\right)$

$\varepsilon \quad$ Relative permittivity

$\theta \quad$ Contact angle $\left({ }^{\circ}\right)$

$\Lambda \quad$ Surface coverage, fraction of surface covered with adsorbed molecules $\left(\mathrm{m}^{2} / \mathrm{m}^{2}\right)$

$\lambda \quad$ Illumination light wavelength (nm)

$\omega \quad$ Uncertainty

\section{Introduction}

Condensation is ubiquitous in many engineering applications and advanced technologies. Examples include power generation (Beér 2007), air conditioning (Daou et al. 2006), and thermal management (Kandlikar and Lu 2009; RahimEsbo et al. 2014). Condensation of vapor can occur in two modes: filmwise and/or dropwise condensation (Tanasawa 1991). It has been shown that the surface heat transfer coefficient is much higher for the dropwise condensation mode (Rose 2002). Dropwise condensation occurs on non-wetting surfaces or partially wetting surfaces (Nouri et al. 2012). After nine decades of research on dropwise condensation (Cho et al. 2017; Emonsi 1939; Enright et al. 2014; Miljkovic and Wang 2013; Schmidt et al. 1930), design and fabrication of a surface that can sustain this mode of condensation for a long term have not been successful (Ahlers et al. 2018; Ma et al. 2019; Oh et al. 2018). The main reason for this is that there is no united understanding of dropwise condensation phenomena because of the complexity of the physics involved. Currently, there are two major theories on the mechanism of dropwise condensation:
1. Film rupture theory: vapor condensates as a thin film on the surface. At a critical thickness, the thin film breaks up and small droplets form on the surface. According to this theory, a thin film (e.g., a film with thickness of one monolayer to several molecule layers) covers the substrate (Jakob 1937; Yongji et al. 1991).

2. Nucleation theory: a droplet nucleates heterogeneously on the surface (Tammann and Boehme 1935). Then, the droplet grows and coalesces to make larger droplets. Heat transfer is dominant through the droplets (defined as the active area); the area between the droplets is considered inactive. Umur and Griffith (1965) showed theoretically there is no film thicker than a monolayer in the inactive area during dropwise condensation.

The inability to accurately define the dropwise condensation mechanism is due to the fact that dropwise condensation occurs at various lengths (from an atomistic order at the early stages of drop formation to mesoscale where body forces are important) and at multiple timescales (from bridge formation between two droplets during coalescence that happens in a micro-second to a quasi-steady state of droplet growth that occurs over several seconds). Therefore, a visualization technique that can shed light on the dropwise condensation mechanism needs to have high spatial and temporal resolutions. Also, the technique should be easy to implement in ambient conditions. Optical microscopy (Chu et al. 2017; Nilsson and Rothstein 2011), and environmental scanning electron microscopy (ESEM) (Anand and Son 2010; Barkay 2013; Rykaczewski et al. 2011; Sharma et al. 2017) are some of the observation techniques that have been reported in the literature for dropwise condensation visualization.

High-speed optical microscopy techniques (Chu et al. 2017; Nilsson and Rothstein 2011) have high temporal resolution. Cha et al. (2016) used top-view mode optical microscopy to observe jumping of a droplet (of a size of hundreds of nanometers) during coalescence at 1000 frames per second (FPS). The lateral resolution of optical microscopy techniques is limited by the diffraction limit. Another drawback of high-speed optical microscopy is its limited ability to measure film thickness. Optical microcopy techniques, such as fringe equal chromatic order interferometry (Zhang et al. 2016a, b), ultrathin film interferometry (Choi et al. 2008), and SPRi (Bayani Ahangar et al. 2019a, b; Kim et al. 2018), can measure film thicknesses less than $1 \mu \mathrm{m}$. Kim et al. used SPRi to provide a 3D map of a thin film with a thickness of less than $400 \mathrm{~nm}$. Still, an optical microscopy technique that can measure sub-nanometer liquid film thicknesses at high speed (>1000 FPS) is not available.

In recent years, ESEM has been used to study a variety of complex interfacial phenomena, such as frost formation (Rykaczewski et al. 2013) and dropwise condensation 
(Zhang et al. 2019). ESEM can provide dynamic information at a length smaller than a micrometer. It is a perfect tool to investigate drop nucleation sites. Recently, phase reconstruction was proposed to enhance the spatial and temporal resolution of ESEM during dropwise condensation experiments (Zhang et al. 2019). Currently, ESEM cannot be employed to study the early stages of coalescence because the temporal resolution of this technique is low (Enright et al. 2014). Moreover, ultrathin film measurement is challenging using ESEM.

Given the lack of direct microscopic techniques with the ability to observe dropwise condensation at multi lengthscales and time-scales, novel experimental methods should be developed to enable better understanding of dropwise condensation. Previously, we reported that SPRi can be implemented to visualize phase change phenomena (Bayani et al. 2018; Jeong et al. 2017; Konduru et al. 2016). The goal of this article is to introduce a newly developed automated SPRi instrument that can be used for quantifying sub-nanometer film at a high temporal resolution of 3000 FPS during dropwise condensation.

\subsection{Surface plasmon resonance imaging}

SPRi is a label-free optical technique that can detect small changes in the refractive index of a dielectric medium near a metal surface (Homola 2003). This method has been used widely to detect biomolecular interactions (Homola 2008). SPRi, or surface plasmon resonance (SPR) microscopy developed from total internal reflection microscopy, was first introduced by Rothenhäusler and Knoll (1988). In principle, SPRi can be used to measure optical properties of dielectric (Bayani Ahangar et al. 2019a, b), film thickness (Kim et al. 2018), and surface coverage (Wolf et al. 2007) on a substrate in a dielectric environment. SPRi has been adapted for studding near-field characterization of fluid flows (Kihm 2010, 2011).

SPR theory can be explained using quantum mechanics and electrodynamics principles. Surface plasmons are oscillations of free electrons at the interface of a dielectric material and a noble metal (such as silver or gold). In SPRi, the surface plasmons are excited optically through the attenuated total internal reflection (ATIR). The most widely used sensor setup for excitation of surface plasmons is based on prism coupling using the Kretschmann configuration; in this setup, incident light comes through the prism and is reflected at the metal layer (Kretschmann and Raether 1968). The electronic excitation results in a drop in the intensity of reflected light. The angle of an incident light at which the maximum drop in the intensity of light occurs is known as the SPR minimum angle.

The intensity of the reflected light depends on several factors, including wavelength, incident angle, polarization, number of mediums above the surface, and refractive index of the mediums. Hence, different SPRi techniques have been developed, such as angular modulation (AM), polarization modulation (PM), wavelength modulation (WM), and intensity modulation (IM). The AM technique has the highest sensitivity for material characterization (Zhang et al. 2016a, b). In the AM approach, the intensity of the light reflected from the dielectric medium/metal interface is measured at a constant wavelength through continuous scanning over the incident angles. The result is an angular spectral curve of the test medium that can be used to find the optimum angle for IM microscopy. Therefore, a high-precision angular microscopy platform is required for SPRi. The biggest challenge for high-precision SPRi with AM is the angle-dependent image artifact (de Bruijn et al. 1993). This artifact, beam walk, arises because the footprint of a beam on the metal substrate spatially transits at each incident angle. Therefore, the surface features move in the recorded images. Beam walk usually occurs in SPRi instruments with an equilateral prism mounted on the rotating stage. As in this SPRi instrument, the prism is rotated to create different incident angles and the beam translation on the surface causes a shift in the position of features inside the beam, and, consequently, in the recorded images.

The conventional AM approach uses a goniometer with a manual rotating stage (Zeng et al. 2017). This technique suffers from three main issues: low precision in angle probing, slow angle-scanning, and angle-dependent image artifacts. To avoid these challenges, automated SPRi instruments have been introduced. Beusink et al. (2008) incorporated a hemispherical prism and an angle-controlled mirror to minimize the beam walk. However, the angle range was small, and it was not suitable for visualization of both liquids and gasses. Ruemmele et al. (2008) presented an inexpensive automated AM platform by mounting a sample/right-angle prism, a focusing lens, and a CCD camera on a single rotating stage. Although their system improved the precision and accelerated the angle-scanning, it could not minimize the beam walk. Zhang et al. (2016a, b) developed an automated SPRi instrument based on AM and IM. In their system, two motorized rotating stages create angular motions of the source light and CCD array. Like prior systems, this system could not eliminate the angle-dependent image artifact. In other research, Zhou et al. (2013) introduced an automated platform for SPRi with AM. This team used a rhombic structure with four connecting arms to convert the linear motion of a piezoceramic motor into the angular motion. Angular probing with high resolution (i.e., $10^{-3 \circ}$ ), was possible with their setup. However, this AM platform could not resolve the issues arising from beam walk.

In this paper, we introduce a newly developed, high-precision angle-scanning SPRi platform that can minimize the angle-dependent image artifact. The developed automated 
SPRi instrument consists of two motorized linear stages and two motorized rotating stages. The light reflected on two angle-controlled mirrors allowed the incident angle to change from $40.0^{\circ}$ to $80.0^{\circ}$, while the linear stages corrected the beam walk by moving the CCD camera and the anglecontrolled mirrors. This new SPRi instrument, capable of imaging with AM and IM, was used for visualization of drop coalescence during dropwise condensation.

\section{Automated SPRi instrument}

A schematic of our automated SPRi instrument is illustrated in Fig. 1. A SugarCube TM Ultra White LED illuminator (Nathaniel Group Inc., Vergennes, VT) is used as a light source. The optics, including an uncoated condenser lens (50-mm diameter, focal length $44 \mathrm{~mm}$, PCX, Edmund Optics, Blackwood, NJ), two iris diaphragms (ID0.5, Newport Optics, Irvine, CA), and an uncoated bi-convex lens (25.4-mm diameter, focal length 100 mm, KBX064, Newport Optics, Irvine, CA), are arrayed to produce a collimated beam with a diameter of $\sim 20 \mathrm{~mm}$. The light passes through a 550-nm hard coated bandpass filter with a full width-half maximum of $10 \mathrm{~nm}$ (Edmund Optics, Blackwood, NJ) and a rotatable polarizer (Thorlabs, Newton, NJ) to create a monochromic p-polarized light beam.

Mirror A is fixed at $135.0^{\circ}$ with respect to the positive direction of the $x$-axis to direct light toward the $z$-direction. Mirror $\mathrm{C}$ is mounted on a motorized rotating stage and can rotate clockwise. This mirror guides light to the prism.
Mirror D is mounted on another motorized linear stage and can spin counter-clockwise. This mirror reflects the light beam vertically toward mirror $\mathrm{B}$, which is fixed at $45.0^{\circ}$ with respect to the positive $x$-axis. Both motorized rotating stages are installed on a linear stage that moves along the $z$-axis. The integrated stages enable mirrors C and D to move in the $z$-axis and spin simultaneously. The SPR sensing system, consisting of a prism-metal test medium, is fixed in space. A Photron APS-RS (PHOTRON USA Inc., San Diego, CA) camera is used to record the reflected light at high speed. The camera is mounted on a linear stage that can move along the $x$-axis. This automated SPRi instrument is controlled by a program written in the Arduino programming language and using an Arduino Mega 2560 Rev3 micro-controller. The same controller board triggers frame acquisition during SPRi with AM.

To minimize beam walk, the $z$ motorized linear stage move according to a motion function. To find the motion function of the $z$ motorized stage, the location of the $z$ stage was adjusted at each incident angle such that the center of the light beam remains stationary at the center of a recorded image during angle-scanning. As the focus of an image at each incident angle affects the intensity of the recorded images and their corresponding reflectance, it is crucial to move the camera during angle-scanning to record focused images. The motion function of the camera's motorized linear stage was determined by adjusting the position of the camera to record focused and crisp images for each incident angle. Hence, the total light path-length of the reflected light from the gold surface to the camera is constant at any

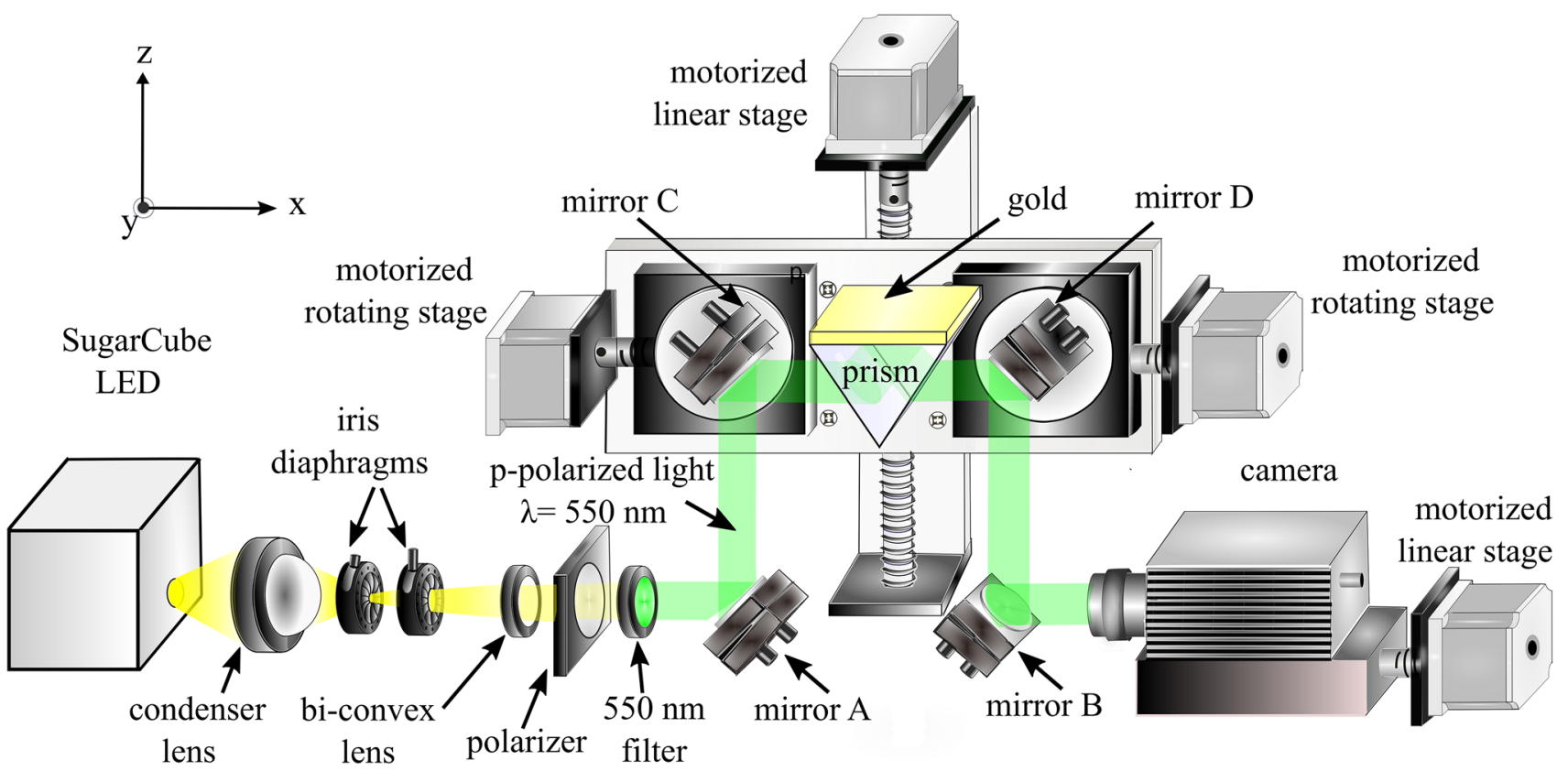

Fig. 1 Schematic of the automated SPRi instrument 
incident angles. Based on the calibration process, the motion functions of all motorized stages were determined and incorporated into the developed Arduino code.

\section{Experimental procedure}

\subsection{Application of the automated SPRi instrument for the condensation/evaporation experiment}

Figure 2 a shows the schematic of the experimental setup. $2.5 \mathrm{~nm}$ of titanium as an adhesion layer and $50 \mathrm{~nm}$ of gold $(n=0.4241+2.4721 i)$ were sputtered (Denton DV6 sputter deposition system) on a glass slide. We utilized atomic force microscopy (AFM) to conduct a roughness characterization of the gold substrate, Fig. 2 b. The roughness characterization was performed on three gold surfaces (three different locations per sample). The roughness average $(\mathrm{Ra})$ on the surface is $1.2 \pm 0.3 \mathrm{~nm}$ and the root mean square of the average roughness $(\mathrm{Rq})$ is $2.2 \pm 0.8 \mathrm{~nm}$. The static contact angle of the gold-coated glass is $79^{\circ} \pm 4^{\circ}$. Before the experiment, the gold-coated glass slide and BK7 prism were cleaned using the standard cleaning protocol (10-min ultrasonic cleaning with isopropyl alcohol 99\% (CAS 67-63-0, Pharmco Aaper), 10-min ultrasonic cleaning in Acetone (CAS 179124-4L-PB, Sigma Aldrich), and vigorous rinsing with DI water for $1 \mathrm{~min}$ ) to remove organic contamination on the sample. The ITOcoated glass was cleaned by sequentially rinsing in IPA, Acetone, and DI water. The gold-coated glass slide was placed on top of the right-angle BK7 prism (size $25.4 \mathrm{~mm}$, $n=1.5185,10 \mathrm{BR} 08$, Newport Optics, Irvine, CA) using a Norland Index Matching Liquid $150(n=1.52$, Norland, USA). SPRi with AM was performed to find the SPR minimum angle of air at $550 \mathrm{~nm}$. To conduct the SPRi with IM, the incident angle was set at $44.8^{\circ}$, which is $1.5^{\circ}$ below the SPR minimum angle of air. Selection of this experimental angle was based on its ability to achieve higher resolution in measurement of thin film on the substrate (see Sect. 4.2). An ITO-coated glass slide was used as a heater and was placed approximately at $5 \mathrm{~mm}$ above the gold surface. A water column (diameter of $\sim 2 \mathrm{~mm}$ and height of $\sim 5 \mathrm{~mm}$ ) was formed between the ITO-coated glass slide and the gold surface. The water column volume was maintained at $15 \pm 5 \mu$ d during the experiment by manually supplying water via a 500- $\mu$ l threaded plunger syringe (Hamilton Company, Reno, NV). Two K-type thermocouples were placed on the gold substrate to monitor the temperature of the gold film. A third K-type thermocouple attached to the syringe needle was placed between the ITO-coated glass and the substrate to measure the air temperature. A sequence of background images was taken at 3000 FPS from the substrate using the Photron APS-RS camera, prior to the onset of dropwise condensation.

Next, the water bridge was heated by connecting the ITO-coated glass to the DC power supply at $12.6 \mathrm{~V}$ and 2.1 A. During the heating process, water evaporates from the water bridge and condensate forms on the gold substrate. ITO-coated glass as a heater was chosen over supplying heated water vapor on the substrate because ITO-coated glass confines dropwise condensation on the gold substrate and ensures that no water film forms on the mirrors.

In our experiments, each CCD camera pixel covered $4 \mu \mathrm{m} \times 4 \mu \mathrm{m}$ of the physical domain at a $550-\mathrm{nm}$ wavelength. The field of view was $4 \mathrm{~mm} \times 6 \mathrm{~mm}$ and the recording speed was 3000 FPS. The temperature of the substrate was $\sim 35^{\circ} \mathrm{C}$, and the temperature of the air was $\sim 45^{\circ} \mathrm{C}$. The temperature of the air is higher than room temperature $\left(24.5^{\circ} \mathrm{C}\right)$, as the heater locally increased the temperature between the ITO-coated glass and the gold substrate. The relative humidity near the substrate where visualization takes place was estimated to be $\sim 60 \pm 4 \%$ using the August-Roche-Magnus formula. Measurement of the temperature of both the air and of the sample when condensation initiated enabled us to determine the relative humidity.

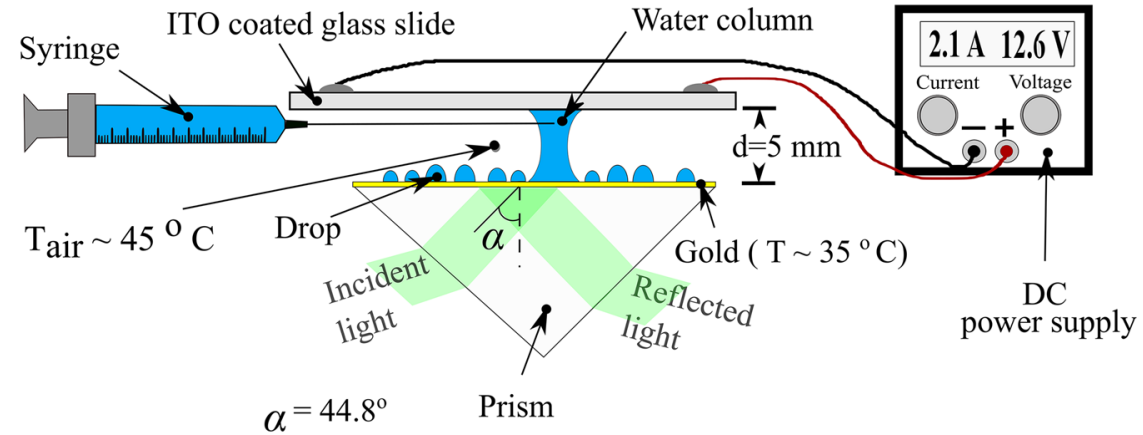

(a)

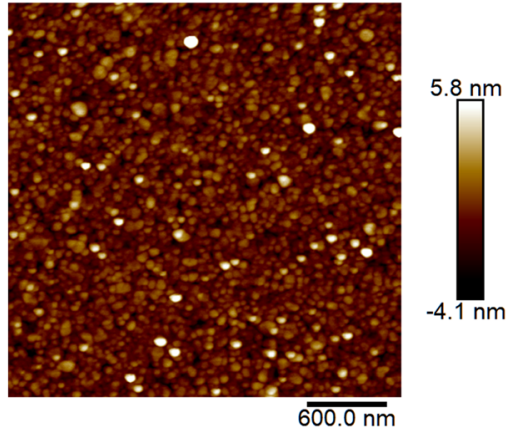

(b)

Fig. 2 a Schematic of the condensation experiment, $\mathbf{b}$ gold substrate roughness measurement by AFM 


\subsection{Image processing method}

Two types of distortion exist in SPR images. First, the images are skewed along one of the axes of the imaging plane due to the oblique incident angle $(\alpha)$. In this work, the skewed images were modified by dividing the vertical axis of the image by $\cos (\alpha)$. Figure 3a, b, respectively, show the raw image and the skewedness-corrected image of the condensation experiment. Because the object plane is not parallel with the image plane in SPRi, there are out-of-focus regions in the images. In the next step of image processing, the stack of background images was averaged over time and subtracted from the time-lapse image of the condensation experiment. This process alleviates the effects of nonhomogeneity in the gold layer or collimated light. The subtracted SPR image was converted into a color index image for easy visualization through a normalization process. The normalized image allows easy comparison between experimental results (Afkhami et al. 2019). The colorful index image can help to decipher details, such as a thin film measurement and material characterization, as compared with the analytical SPR results. To normalize the image and calculate the reflectance, a calibration factor is defined as $S=\left(I_{\max }-I_{\min }\right) /\left(\operatorname{Ref}_{\max }-\operatorname{Ref}_{\min }\right)$, where $I_{\max }$ and $I_{\min }$ represent maximum and minimum intensity from SPRi with AM. $\operatorname{Ref}_{\max }$ and $\operatorname{Ref}_{\text {min }}$ indicate the theoretical maximum and minimum values of reflectance of air at $550 \mathrm{~nm}$ (Fig. 5). Using a calibration factor, we can convert the pixel intensity value to the unit of reflectivity ( $0-1)$. A detailed description of the reflectance calculation and image processing method used in this work can be found in our recent paper (Bayani et al. 2018). The normalized image with the reflectance unit is shown in Fig. 3c. The yellow color with the reflectance of 0.594 represents the bulk liquid on the gold substrate; the blue color with the index of 0.347 shows the presence of air/ water vapor on the substrate. Our SPRi instrument cannot differentiate the gasses (the difference in refractive index of gasses in ambient conditions is on the order of $1 \mathrm{e}-4$, which is beyond the capability of our SPRi instrument to measure refractive index); thus, we can be sure that gas (air/water vapor) exists on the surface. Due to high-speed imaging (low light), the noise level is high in the image. Therefore, the reflectance at each pixel of the background image fluctuated around 0.347 .

\subsection{Noise analysis in high-speed SPRi}

One of the main goals of this article is to show the feasibility of measuring sub-nanometer film at high-speed temporal resolution. To report measured values of thickness with high confidence, the error associated with the measurement is required to be some fraction of the thickness being measured. In measuring techniques based on imaging, noise causes imprecision in measurement of pixel intensity values. This section details the noise analysis method used in highspeed SPRi. The noise analysis result is used to find the error of the thickness measurement (see Sect. 4.2).

Noise in the signal has a stochastic nature; in addition, it adds a random unwanted fluctuation to the signal. Random fluctuation in the signal is difficult to identify as the measurement of a signal at one time, and one location, does not provide information about the next or previous frame. Noise in the SPRi is produced by factors such as instability in the illumination light, nonhomogeneous light, vibration, shot noise, sensor temperature, lens distortion, and digitization hardware. The systematic noise, like the noise created due to nonhomogeneous light, is reduced during image processing by subtracting the background image from the condensation image. The remaining noise in the signal can be characterized in two ways-Gaussian noise and signaldependent noise. The Gaussian noise is independent of signal intensity; its value at each pixel is calculated by an independent draw from a Gaussian probability distribution. The signal-dependent noise, including the shot noise (i.e., fluctuations of the number of photons detected at a given exposure level), the fluctuation in light, and the vibration,

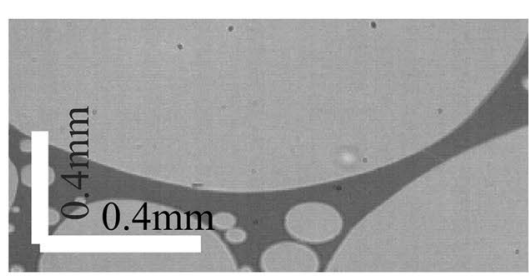

(a)

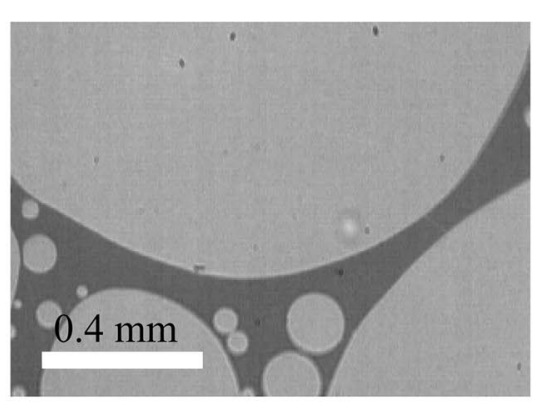

(b)

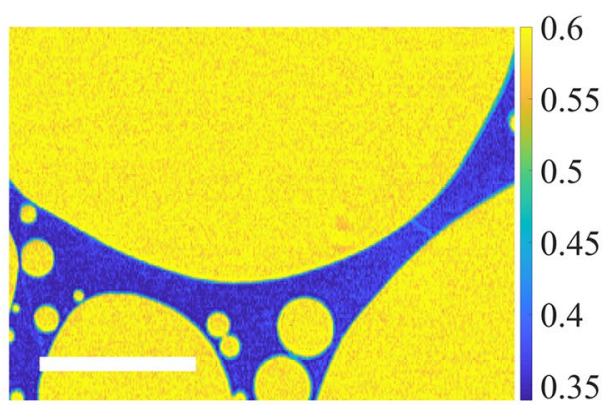

(c)

Fig. 3 a Raw image, $\mathbf{b}$ a skewedness-corrected SPR image derived by dividing the vertical axis of the image plane by $\cos (\alpha)$, $\mathbf{c}$ normalized SPR images with the reflectance unit 
follows a Poisson distribution. In practice, signal-dependent noise has the lower bound on the uncertainty of measuring light; further, noise in the imaging can be modeled using a Gaussian distribution (Hasinoff 2014). A simple technique to reduce the Gaussian noise is to calculate the average value of the neighbors of a pixel. In this method, each pixel output is the mean value of its kernel window.

Image noise was analyzed by picking a pixel randomly and calculating the mean values of the signal over the pixel neighborhoods of $1 \times 1,3 \times 3,5 \times 5,9 \times 9$, and $17 \times 17$ at each frame. The same procedure was repeated at ten random locations of the image. Figure 4 demonstrates the reflectance of the pixel at kernel windows of $1 \times 1,5 \times 5,9 \times 9$ and $17 \times 17$ pixels. At each kernel window, two plots are illustrated: (1) signal (reflectance) fluctuation during the time-lapse imaging, and (2) histogram of the signal. The total number of time-lapse images is 8000 and each frame takes $0.33 \mathrm{~ms}$. The histogram at all the kernel windows can be fitted with a Gaussian distribution. The result verifies the assumption that behavior of noise in high-speed SPRi can be described with a Gaussian distribution. The signal-noise ratio and the coefficient of variation are calculated from the noise analysis result (see Table 1). These values are used to quantitatively estimate the probability of noise during high-speed imaging of condensation. Signal-noise ratio-the mean of the signal over its standard deviation-increases from $39 \pm 4$ to $307 \pm 23$ when the kernel window changes from $1 \times 1$ to $17 \times 17$. The higher signal-noise ratio implies less noise in the reflectance measurement. Coefficient variation-the ratio of standard deviation over the mean value of a signalrepresents the dispersion of a signal around the mean value. Using a kernel window of $5 \times 5$, the probability of noise at each pixel is reduced to \pm 0.0057 of the signal value.

We employed an averaging filter with pixel neighboring of $5 \times 5$ to the normalized images (Sect. 4.2). This size of filter confines the noise at each pixel to \pm 0.0057 of the signal values, while not sacrificing details of the image due to a blurring effect.
Table 1 Signal-noise ratio and coefficient of variation of the reflectance signal at different kernel windows

\begin{tabular}{llc}
\hline Kernel windows & Signal-noise ratio & Coefficient of variation \\
\hline $1 \times 1$ & $39 \pm 4$ & $0.026 \pm 0.003$ \\
$3 \times 3$ & $104 \pm 12$ & $0.0093 \pm 0.0008$ \\
$5 \times 5$ & $163 \pm 6$ & $0.0057 \pm 0.0007$ \\
$9 \times 9$ & $228 \pm 23$ & $0.0044 \pm 0.0004$ \\
$17 \times 17$ & $307 \pm 23$ & $0.0032 \pm 0.0002$ \\
\hline
\end{tabular}

\subsection{Uncertainty evaluation for SPR reflectance}

Uncertainty analysis was conducted based on single point detection estimation (Kim et al. 2018; Kim and Kihm 2006). The resulting reflectance can be calculated from $R_{\mathrm{SPR}}=f\left(\varepsilon_{1}, \varepsilon_{2}, \varepsilon_{3}, \varepsilon_{4}, d_{2}, \lambda_{i}, \alpha, d_{3}\right)$, where subscripts 1,2 , 3 , and 4 refer to the BK7 prism, gold, water, and air. The uncertainty of reflectance can be evaluated as:

$\omega_{R_{\mathrm{SPR}}}= \pm\left[\begin{array}{l}\left(\frac{\partial R_{\mathrm{SPR}}}{\partial \varepsilon_{1}} \omega_{\varepsilon_{1}}\right)^{2}+\left(\frac{\partial R_{\mathrm{SPR}}}{\partial \varepsilon_{2}} \omega_{\varepsilon_{2}}\right)^{2}+\left(\frac{\partial R_{\mathrm{SPR}}}{\partial \varepsilon_{3}} \omega_{\varepsilon_{3}}\right)^{2}+\left(\frac{\partial R_{\mathrm{SPR}}}{\partial \varepsilon_{4}} \omega_{\varepsilon_{4}}\right)^{2} \\ +\left(\frac{\partial R_{\mathrm{SPR}}}{\partial d_{2}} \omega_{d_{2}}\right)^{2}+\left(\frac{\partial R_{\mathrm{SPR}}}{\partial \lambda} \omega_{\lambda}\right)^{2}+\left(\frac{\partial R_{\mathrm{SPR}}}{\partial \alpha} \omega_{\alpha}\right)^{2}+\left(\frac{\partial R_{\mathrm{SPR}}}{\partial d_{3}} \omega_{d_{3}}\right)^{2}\end{array}\right]^{1 / 2}$,

where the elementary uncertainties are estimated as $\omega_{\varepsilon_{1}}=0.01 \% \times \varepsilon_{1}, \omega_{\varepsilon_{2}}=0.1 \% \times \varepsilon_{2}, \omega_{\varepsilon_{3}}=0.01 \% \times \varepsilon_{3}$, $\omega_{\varepsilon_{4}}=0.01 \% \times \varepsilon_{4}, \omega_{d_{2}}=0.1 \% \times d_{2}, \omega_{\lambda}= \pm 5 \mathrm{~nm}$, $\omega_{\alpha}=0.2^{\circ}$ (beam divergence), and $\omega_{d_{3}}= \pm 0.125 \mathrm{~nm}$ (uncertainty of a monolayer thickness measurement). The uncertainties of the prism, water, and air are assumed to be negligible because of their small variation of $0.01 \%$. The uncertainty of reflectance at $0 \mathrm{~nm}$ and monolayer thickness of thin water film is close to \pm 0.07 . All the variables are taken at the test condition $\left(\varepsilon_{2}=-5.931+2.097 i\right.$, $d_{2}=50 \mathrm{~nm}, \lambda=550 \mathrm{~nm}$, and $\alpha=44.8^{\circ}$ ). The effect of beam divergence has the maximum weight on the estimated uncertainty for the reflectance measurement when considering only the reflectivity. Under this restriction, the estimated

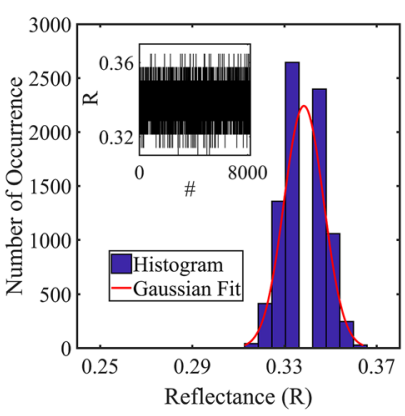

(a)

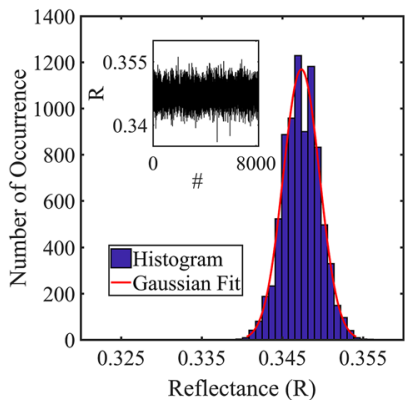

(b)

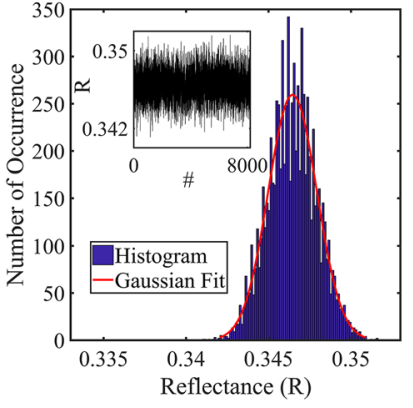

(c)

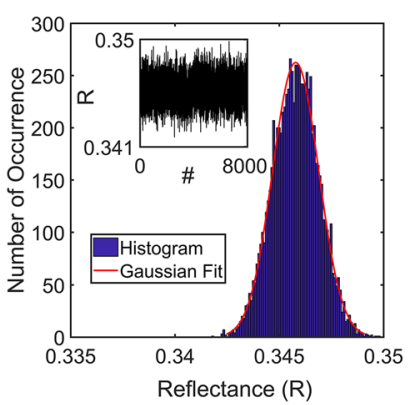

(d)

Fig. 4 Analysis of SPR image noise - at an experimental angle of $44.8^{\circ}$, a temporal resolution of $3000 \mathrm{FPS}$, and a spatial resolution of $4 \mu \mathrm{m}-$ by averaging the reflectance signals over kernel windows of $\mathbf{a} 1 \times 1$ pixels, $\mathbf{b} 5 \times 5$ pixels, $\mathbf{c} 9 \times 9$ pixels, $\mathbf{d} 17 \times 17$ pixels; \# stands for frame number 
reflectance uncertainty is larger than the monolayer thickness measurement. However, additional factors need to be considered when discussing the uncertainty in the thickness measurement. First, the intensity value of the background image is subtracted from the pixel intensity value of the time-lapse image of condensation. This process reduces the effect of beam divergence on the reflectance uncertainty. For example, when the thickness of the water film is zero on the gold surface, the reflectance $\left(\lambda=550 \mathrm{~nm}\right.$ and $\left.\alpha=44.8^{\circ}\right)$ of the pixel is $0.347 \pm 0.03$. The error in measured reflectance is less than estimated uncertainty using Eq. (1). Second, we are using the concept of pixel neighboring to reduce the error of the reflectance measurement at a single pixel when measuring the reflectance of the thin film and computing the corresponding film thickness. Third, the field of view in our work is located approximately at the center of the incident beam on the BK7-gold interface. The size of the field of view is $4 \mathrm{~mm} \times 6 \mathrm{~mm}$ which is only $\sim 7 \%$ of the cross-sectional area of the beam. As a result, the net effect of beam divergence on the reflectance uncertainty is much less than the estimated value, based on the total prism area.

\section{Results and discussion}

This section first lists the advantages of automated SPRi in high-speed visualization of phase change phenomena. The discussion includes advancement of SPRi with AM, and ambient-temperature-independent reflectance measurement. Then, the results of visualization of dropwise condensation are discussed and compared with theories of the dropwise condensation mechanism.

\subsection{Advantages of automated SPRi in visualization of phase change phenomena}

\subsubsection{Advancement of SPRi with AM}

One critical challenge in angular SPRi is the fine adjustment of incident light, optical arrays, the prism, and the CCD camera at each incident angle. Operator skill during angle-scanning can cause large uncertainty in SPRi with AM. On the other hand, the process of angular tuning of the setup requires meticulous care and is time-consuming. Our newly developed automated SPRi platform resolved these issues associated with conventional angular SPRi. The user can define the angular increment for the scanning from $10^{-30}$ to $1.0^{\circ}$ easily through the controlling program. Each increment takes about $3 \mathrm{~s}$. Depending on the desired precision and the angle range, the time required for angular scanning is different. Figure 5 represents the theoretical and experimental reflectance curve of air at $550 \mathrm{~nm}$. Details about the approach used in this work to find the theoretical reflectance can be found in Yamamoto

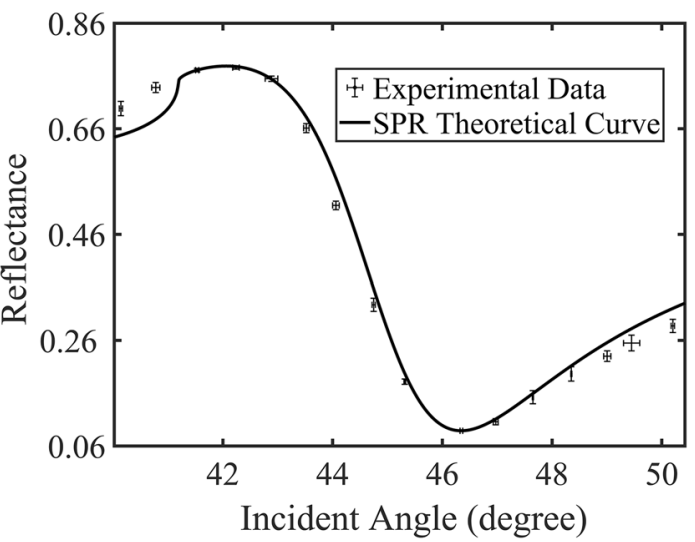

Fig. 5 Theoretical and experimental SPR reflectance comparison of air at $550 \mathrm{~nm}$ (the error bar represents the deviation in the measurement based on 5 data sets)

(2002). The error bar shows the experimental results for 5 different sets of experiments. Each experiment took approximately 1 min to complete. The SPR signal behavior perfectly matches with the theoretical result around the maximum and minimum points of the curve. The deviation from the theoretical result increases between these two probing angles or at an angle larger than the SPR minimum. The maximum discrepancy between the experimental and the theoretical results is at the incident angles smaller than the internal reflection angle $\left(41.2^{\circ}\right)$. The experimental reflectance curve is in closer agreement with the theoretical reflectance curve when a smaller angle step is selected for the SPRi with AM.

As Fig. 5 shows, small changes in the incident angle can cause a significant change in the reflectance. Hence, knowing the exact position of the incident angle is crucial for analysis related to SPRi with IM. Having motorized rotating mirrors enabled us to adjust the incident angle by minimizing human error that may occur when using a manual SPRi instrument. Moreover, once the incident angle was fixed, we double-checked the position by experimentally measuring the reflectance of air and water (bulk) at the fixed angle and wavelength. The reflectance was measured by dividing the intensity of light in the p-polarized mode by the intensity of light in the s-polarized mode.

\subsubsection{Ambient-temperature-independent reflectance measurement}

Condensation experiments are usually performed using a Peltier stage, which induces a fixed temperature on the surface. However, our experiments were not conducted using a Peltier stage, and the temperature of the gold substrate was recorded during the experiment. SPR reflectance can be affected by temperature changes (Chiang et al. 2007; Kim and Kihm 2007). Therefore, it was imperative to consider 
the temperature-dependent behavior of SPR when we were analyzing the recorded images. Theoretical modeling and experimental investigation were implemented to evaluate the effect of temperature. SPR reflectance depends on the optical properties of the BK7 prism, the gold, and the test medium (air or water). It has been shown that the refractive index of the BK7 prism only changes 0.0002 when temperature changes from 25 to $125{ }^{\circ} \mathrm{C}$ (Englert et al. 2014). Thus, in this work, we can neglect the effect of the BK7 prism as the temperature on the gold changed only from 25 to $35^{\circ} \mathrm{C}$. According to the Lorenz-Drude temperature model (Alabastri et al. 2013), temperature variation can affect the optical properties of gold by changing the plasmon frequency and the electron collision rate. This model was used to simulate the effect of temperature on the gold's optical properties.

The air and water refractive indices decrease with an increase in temperature. The sensitivity of our SPRi instrument in measuring the refractive index was less than the refractive index changes of the air. Therefore, we can consider the temperature-independent behavior for air. The change of the refractive index of water as a function of temperature was modeled using an empirical relationship (Bashkatov and Genina 2003). Figure 6a shows the SPR reflectance curve for air and water at $550 \mathrm{~nm}$ when the temperature changes from 25 to $75^{\circ} \mathrm{C}$. The model predicted that the SPR curve of air would shift slightly to the right with an increase in temperature. At the SPR minimum angle of air at $550 \mathrm{~nm}$, the air reflectance changes less than $1 \%$ with a $50{ }^{\circ} \mathrm{C}$ increase in temperature. Simulation of the water reflectance curve at $550 \mathrm{~nm}$ indicated that the water reflectance curve drastically changes with rises in temperature; however, the reflectance variation is less than $1 \%$ when the incident angle is between $30.0^{\circ}$ and $55.0^{\circ}$ - we performed the condensation visualization at $44.8^{\circ}$. Also, we experimentally depicted the
SPR signal behavior with the temperature changes at a probing angle of $46.3^{\circ}$ and a wavelength of $550 \mathrm{~nm}$ (see Fig. 6b). The reflectance of the SPR signal was measured using the same method discussed in the image processing section. The temperature on the $x$-axis represents the temperature of the gold substrate. The air temperature is $\sim 20^{\circ} \mathrm{C}$ more than the substrate and the water temperature is $\sim 10{ }^{\circ} \mathrm{C}$ more than the substrate temperature. The reflectance changes represent the accumulative dependence of the SPR sensor, including the BK7 prism, the gold substrate, and the index matching liquid. The result indicates that the reflectance variation is less than $1 \%$ when the gold temperature varies from 25 to $70{ }^{\circ} \mathrm{C}$, the incident angle is $46.3^{\circ}$, and the wavelength is $550 \mathrm{~nm}$. Both experimental and theoretical results show that the change in reflectance is less than $1 \%$.

In summary, the nearly temperature-independent behavior of the SPR signal close to the SPR minimum angle of air allows visualization of the condensation/evaporation process without a thermally controlled test chamber between 25 and $70{ }^{\circ} \mathrm{C}$. This means by carefully selecting the probing angle, the phase change phenomena visualization experiment can be carried out in ambient conditions. This significantly reduces the costs of the experiment. In the dropwise condensation experiment (see Fig. 2), the temperature of the air is $\sim 45^{\circ} \mathrm{C}$ and the temperature of the gold is $\sim 35^{\circ} \mathrm{C}$; this is in the range $\left(25-70{ }^{\circ} \mathrm{C}\right)$ of the temperature-independent behavior that is shown. Hence, it can be concluded that the results of the dropwise condensation experiment are not affected by changes in temperature. It is possible to use the SPRi technique in the case of higher-temperature ambient conditions (such as condensation of steam at $100{ }^{\circ} \mathrm{C}$ ) if the incident angle and wavelength of light are chosen wisely. However, surface degradation needs to be studied when working with steam at $100{ }^{\circ} \mathrm{C}$. For example, volatile organic compounds

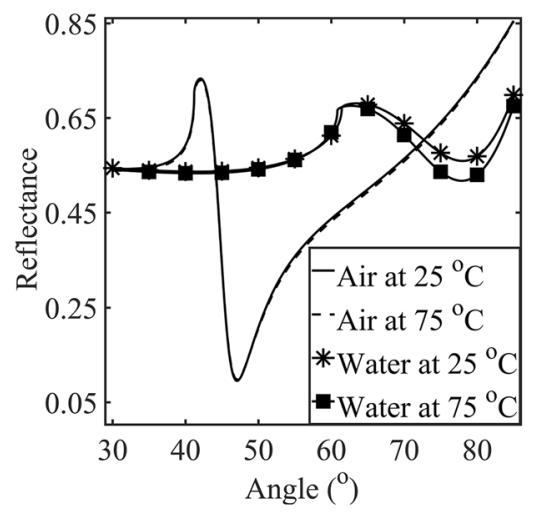

(a)

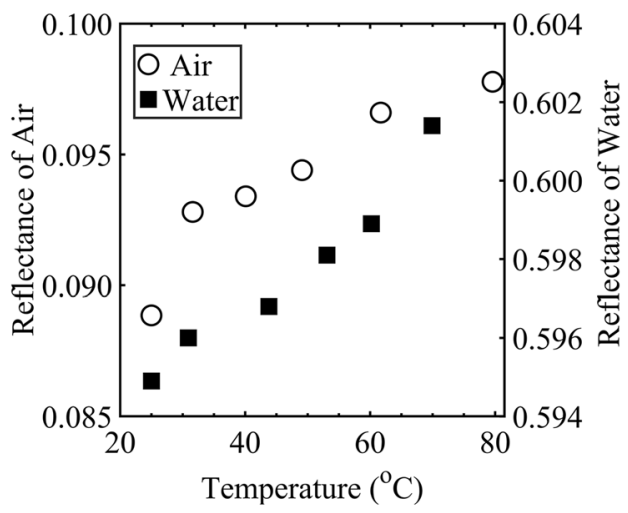

(b)

Fig. 6 Temperature-dependent behaviors of the SPR signals in air and water environments; a the theoretical model shows the change in reflectance at a probing angle of $46.3^{\circ}$ and wavelength of $550 \mathrm{~nm}$ is less than $1 \%$; $\mathbf{b}$ the experimental data indicates that at a fixed angle of

$46.3^{\circ}$ and wavelength of $550 \mathrm{~nm}$, the changes in reflectance are less than $1 \%$ for both air and water when the temperature of the gold substrate changes from 25 to $70{ }^{\circ} \mathrm{C}$ (temperature of air and water are, respectively, $\sim 20^{\circ} \mathrm{C}$ and $\sim 10^{\circ} \mathrm{C}$ higher than the substrate) 
may desorb at higher temperatures and affect the wettability of the surface as well as the water molecules' adsorption kinetics.

\subsection{Dropwise condensation}

The dropwise condensation process begins by nucleation of droplets on the substrate and subsequent growth of these droplets without significant interactions. In the next stage of dropwise condensation, two or more adjacent droplets coalesce and produce a larger composite droplet. Droplet motion due to coalescence exposes part of the substrate to the ambient air; then, the nucleation process is repeated at the exposed area. This condensation cycle continues on the substrate. In this section, we shed light on the dropwise condensation mechanism by studying the fluid-solid interface during coalescence phenomena.

It has been shown that volatile organic compounds can absorb rapidly on the surface and change wettability of the metal substrates (Cha et al. 2017; Orejon et al. 2019; Preston et al. 2014; Yan et al. 2019a, b). Gold that is intrinsically hydrophilic can support dropwise condensation when it is in the ambient atmosphere (Umur and Griffith 1965). In this work, since low condensation rates were implemented to conduct the experiment, condensation on the gold-coated glass remained dropwise during the experiment. There is an initial transient in condensation that reaches a stationary rate of approximately $0.0009 \mu \mathrm{l} /\left(\mathrm{mm}^{2} \mathrm{~s}\right)$, or a heat transfer rate of $0.2 \mathrm{~W} / \mathrm{cm}^{2}$; this rate corresponds to relative humidity of $60 \%$. The droplet growth rate is estimated to be on the order of $1 \mu \mathrm{m}$. The droplet growth rate and heat transfer rate were quite low mainly due to the presence of non-condensable gases (Chavan et al. 2016; Enright et al. 2014). Figure 7 illustrates the evolution of coalescence on a gold substrate. Filter averaging of $5 \times 5$ was applied on the normalized images to reduce the image noise. The initial status of the drop before coalescence is depicted in Fig. 7a. The yellow color shows the droplet and the blue color represents the area between droplets (from here on, it is called an inactive area). During the coalescence, the involved droplets move on the substrate, which exposes part of the surface to the water vapor in the ambient air. From here on, the exposed area after the coalescence is called a preoccupied area. Figure $7 \mathrm{~b}$ illustrates the preoccupied area after $9 \mathrm{~ms}$ from the start of coalescence. After the coalescence event, new small droplets form on the preoccupied area, as shown in Fig. 7c.

It can be observed from Fig. $7 \mathrm{~b}$ that the preoccupied area has a slightly different color from the inactive area. For easy visualization, the contrast of this image has been enhanced by changing the range of the color map (see Fig. 8a). To compare the reflectance of the inactive and preoccupied areas, the averaged reflectance of each site was calculated. The preoccupied area with 27,500 pixels has the averaged reflectance of $0.34582 \pm 1.2 \mathrm{E}-5$, which is very close to the reflectance of a gold-air interface. The standard error (i.e., $\pm 1.2 \mathrm{E}-5$ ) is measured by dividing the standard deviation of noise at each pixel (i.e., 0.0057 of the signal value) by the root mean square of the number of observations, 27,500. The averaged reflectance of the inactive area with 15,000 pixels is $0.35401 \pm 1.6 \mathrm{E}-5$. The difference of reflectance between the preoccupied and the inactive areas is 0.00819 , which is more significant than the standard errors of the measurement. The difference in the reflectance values at the two sites indicates there exists a material difference between the preoccupied area and the inactive area. SPR reflectance at the fixed angle and wavelength depends on the refractive indices and the thickness of the test media involved. As the refractive index of air and water is constant over the entire study area, the difference of reflectance values can only be attributed to the changes in the thickness of the water film.

Figure $8 \mathrm{~b}$ depicts the theoretical SPR curve at $550 \mathrm{~nm}$ for a 4-layer medium (BK7 prism, gold, thin water film, and air) with different thicknesses of water film. It can be seen in the figure that the sensitivity in measuring thin film

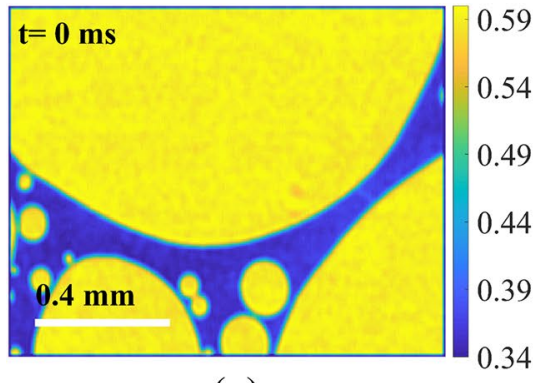

(a)

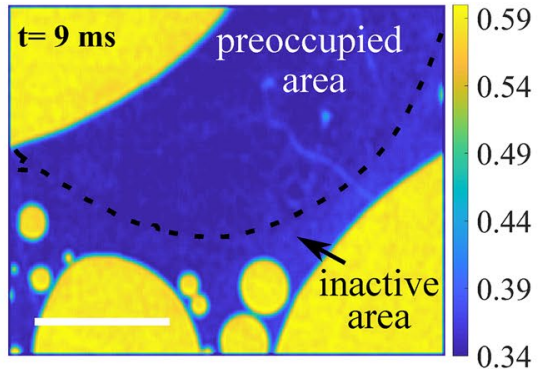

(b)

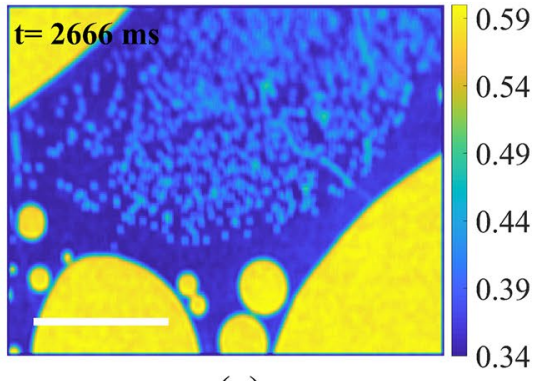

(c)
Fig. 7 Coalescence evolution: a initial positions of droplets before coalescence; the yellow color represents water and the blue color signifies air; $\mathbf{b}$ an exposed part of the substrate after the droplet motion during coalescence-the exposed area (preoccupied area) is distinct with a slightly different color index, as compared to the ambient (inactive area) result; $\mathbf{c}$ nucleation of new droplets in the preoccupied area only 


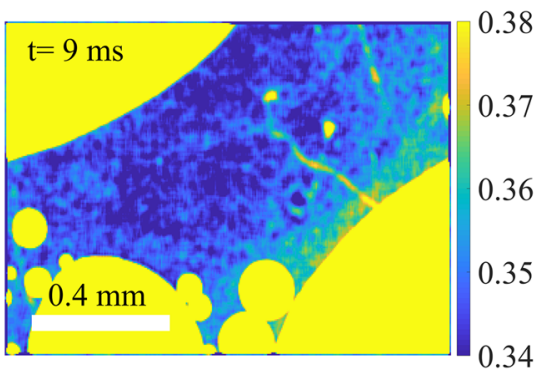

(a)

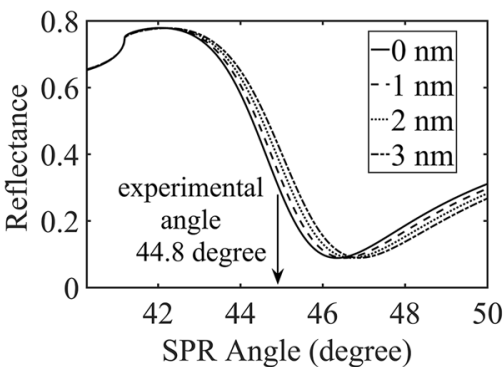

(b)

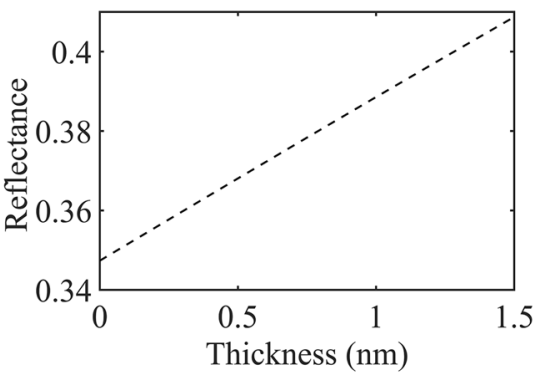

(c)
Fig. 8 a Reflectance of the inactive area is 0.00819 larger than preoccupied area with reflectance of 0.34582 ; $\mathbf{b}$ theoretical SPR curve for the 4-layer medium (BK7 prism, gold layer, thin water film, and air) with different sizes of water film at $550 \mathrm{~nm}$; $\mathbf{c}$ the change of reflectance with the increase in the size of the water film at $44.8^{\circ}$; the reflectance of the preoccupied are represents the dry area on the substrate and the reflectance of the inactive area shows the existence of a monolayer film between droplets is higher when SPRi with IM is performed at $1.0^{\circ}-3.0^{\circ}$, far from the SPR minimum angle of air; that is the reason that $44.8^{\circ}$ was selected as the experimental angle for SPRi with IM. At this angle, a monolayer thickness of water film $(0.275 \mathrm{~nm})$ yields a 0.01150 increase in the SPR reflectance value (see Fig. 8c). As the reflectance of the preoccupied area is very close to the reflectance of the gold-air interface, it can be concluded that only air exists on the gold surface, i.e., the preoccupied area is a dry zone. However, the reflectance of the inactive area is 0.00819 larger than the reflectance of the gold-air interface and the 0.00819 of reflectance unit indicates the presence of an adsorbed water film (neither high-concentration water vapor nor adsorbed volatile organic compounds) with an average thickness of less than a monolayer at the inactive area. The average thickness of less than a monolayer means that the adsorbed film is not covered uniformly over the entire area between droplets (see Sect. 4.2.1). It should be mentioned that it is likely that droplets constantly evaporate on the surface due to the high temperature of the substrate $\left(\sim 35^{\circ} \mathrm{C}\right)$; this may cause a high concentration of water vapor around the droplets. However, the automated SPRi instrument cannot differentiate the difference between gasses, i.e., the sensitivity of the SPRi instrument in measuring the refractive index is less than the change of refractive indices from air to high-concentration water vapor.

The aforementioned standard cleaning protocol using isopropyl, acetone and DI water cannot remove the adsorbed volatile organic compounds from the surface (Cha et al. 2017; Saga and Hattori 2005; Yan et al. 2019a, b). It was likely that adsorbed organic compounds were present on the surface even prior to the visualization experiment. Moreover, the preoccupied area that emerges after the coalescence is dry, i.e., it does not have the adsorbed water thin film. It has been reported that DI water cannot remove adsorbed organic compounds on the surface (Saga and Hattori 2005). This means that if an organic adsorbed film exists on the surface, it cannot be removed by the droplet. As the reflectance measurement was conducted by subtracting the pixel value of the background images from condensation mages, the net effect of adsorbed volatile organic compounds on the reflectance would be zero or negligible. Therefore, the changes in reflectance cannot be attributed to the adsorption of volatile organic compounds on the surface.

The nucleation of droplets at the preoccupied area can be explained with the new experimental results. The adsorption of vapor molecules on the surface minimizes the surface energy of the substrate. As the preoccupied area is dry, it has higher surface energy compared to the inactive area between the droplets. The preoccupied area with high surface energy serves as a favorable site for water vapor to condense and nucleate on the substrate, as shown in Fig. 7c. We cannot comment on the evolution of the dry zone between $9 \mathrm{~ms}$ and the time when nucleation starts; this is because the spatial resolution $(4 \mu \mathrm{m})$ is larger than the size of the first nucleus. In our future work, we will answer to the question of whether an adsorbed film forms on the dry zone, followed by nucleation or whether the vice versa scenario occurs.

\subsubsection{Mechanism of dropwise condensation}

There is no united understanding of dropwise condensation phenomena because of the complexity of the physics involved. The mechanism of dropwise condensation has been under debate for the past 90 years (Leipertz 2010). Two main theories have emerged; both theories have been supported with experimental results (Umur and Griffith 1965; Yongji et al. 1991). However, the experimental methods used in these studies were not able to measure sub-nanometer film thicknesses at high speeds ( $>1000$ FPS) in ambient conditions. To the knowledge of the authors, this is the first time that the coexistence of an adsorbed film between a droplet and the dry zone at the preoccupied area during dropwise condensation has been experimentally reported. 
Although the film rupture theory can explain the existence of a thin film between the droplets, it is unable to explain the existence of a dry zone at the preoccupied areas. Thus, the results of this study undermine the validity of film rupture theory. Our results suggest that either nucleation theory or a combination of both film rupture and nucleation theory can explain dropwise condensation on a solid substrate.

The average thickness of the absorbed layer between droplets presented here can be explained with the theoretical approach that Umur and Griffith (1965) used in their work. According to the adsorption theory, molecules in the gas can adhere to the solid surface. The fraction of a surface covered by adsorbed molecules is called surface coverage. Langmuir theory (Langmuir 1933) explains that striking molecules on the surface can adsorb them to the surface; subsequently, they evaporate from the surface. Under an equilibrium condition, the rate of adsorption is equal to the rate of evaporation. The Langmuir isothermal model assumes the maximum surface coverage of the adsorbed molecule is one monolayer. The rate of adsorption on a surface and evaporation from the surface, respectively, can be expressed as:

$r_{\mathrm{a}}=k_{\mathrm{a}} \frac{P}{\sqrt{2 \pi m K_{\mathrm{B}} T}}$,

$r_{\mathrm{d}}=k_{0} \Lambda e^{\frac{-w}{R T}}$

To find the surface coverage during dropwise condensation, consider a solid substrate that has interface with both vapor and liquid under an equilibrium condition ( $T$ and $P$ are constant). For this system, we can write at the solid-vapor interface (subscript s) and liquid-vapor interface (subscript 1), respectively:

$$
\begin{aligned}
& k_{\mathrm{as}} \frac{P}{\sqrt{2 \pi m K_{\mathrm{B}} T}}=k_{0} \Lambda_{\mathrm{s}} e^{\frac{-w_{\mathrm{S}}}{R T}}, \\
& k_{\mathrm{al}} \frac{P}{\sqrt{2 \pi m K_{\mathrm{B}} T}}=k_{0} \Lambda_{1} e^{\frac{-w_{l}}{R T}}
\end{aligned}
$$

For the liquid interface, $\Lambda_{l}$ is one. By dividing Eq. (4) by Eq. (5), the $\Lambda_{s}$ can be expressed as:

$$
\Lambda_{\mathrm{s}}=\frac{k_{\mathrm{as}}}{k_{\mathrm{al}}} e^{\frac{w_{l}}{R T}\left(\frac{w_{s}}{w_{l}}-1\right)}
$$

The values for $k_{\text {as }}$ and $k_{\text {al }}$ are close to unity; therefore, their ratio can be considered to be one. It can be shown that the work of adhesion between the solid-liquid interface to the work of cohesion between the liquid-liquid interface is (Umur and Griffith 1963); $\frac{w_{\mathrm{s}}}{w_{1}} \approx \frac{1}{2}(1+\cos \theta)$

For the case of dropwise condensation where $\theta$ is larger than zero, the $w_{\mathrm{s}} / w_{1}$ is smaller than one. Using the properties of water at the pressure of 1 bar and temperature of $35{ }^{\circ} \mathrm{C}$ and considering molar latent heat of $43.56 \mathrm{~kJ} / \mathrm{mol}$ instead of $w_{\mathrm{l}}$, one obtains $\Lambda_{\mathrm{s}}<1\left(\mathrm{~m}^{2} / \mathrm{m}^{2}\right)$. The average thickness of the surface is proportional to the surface coverage. Surface coverage in our experiment can be obtained to be $0.71\left(\mathrm{~m}^{2} / \mathrm{m}^{2}\right)$. Our measurement shows the thickness of the adsorbed layer is not greater than a monolayer in majority of the inactive area. However, based on our visualization, there are some limited locations on the inactive area, such as the area between two large droplets (see Fig. 7a) that have a local thickness of more than one monolayer. We are not certain whether these areas have adsorbed film more than a monolayer or if they are covered with micro/nano droplets less than the spatial resolution of our imaging $(4 \mu \mathrm{m})$. In either case, the effect of these areas is neglected in the heat transfer models developed based on the nucleation theory of dropwise condensation (Khandekar and Muralidhar 2014). It is possible to further improve the spatial resolution of SPRi (Kim and Kihm 2006). In our future studies, we will enhance the spatial resolution $(550 \mathrm{~nm})$ of the SPRi instrument to study these high-density areas on the substrate, and interaction of water molecules in the adsorbed layer with neighboring droplets. Moreover, the mechanism of initial drop formation at the onset of condensation will be studied to enable a full view of the dropwise condensation mechanism on the solid substrate.

\section{Conclusion}

The current study developed an automated, angle-scanning SPRi instrument by integrating linear and rotating motorized stages. This instrument improves the conventional SPRi that uses the AM technique by enhancing the resolution of angle probing, increasing the speed of angle-scanning, and minimizing the angle-dependent image artifact. This automated instrument can perform angle-scanning at a range of $40.0^{\circ}-80.0^{\circ}$, which is suitable for sensing a variety of organic dielectrics, including air and water. The angular resolution can easily be adjusted from $10^{-3}$ to $1.0^{\circ}$, depending on the precision required for an experiment. The instrument has a large field of view, which is perfect for studies related to fluid dynamics applications. The SPRi instrument, combined with high-speed imaging, introduces a new tool for real-time imaging of phase change phenomena, such as drop coalescence, condensation, and evaporation. Our newly developed, automated SPRi instrument successfully recorded the evolution of drop coalescence at 3000 FPS and 
a spatial resolution of $4 \mu \mathrm{m}$. This SPRi instrument will be able to achieve higher spatial and temporal resolutions when a stronger illumination system and a larger NA objective lens are implemented for the imaging. We numerically and theoretically demonstrated that the SPR signal dependence on temperature close to the SPR minimum angle of air is less than $1 \%$ when the temperature changes from 25 to $70{ }^{\circ} \mathrm{C}$. This means that there is no need for a thermally controlled test chamber or a Peltier stage for visualization of phase change phenomena. The probability of noise in the imaging was studied and used to measure the standard error of the thin film measurement. Our observation of dropwise condensation reveals (1) the presence of a thin film with thickness of one monolayer, and (2) surface coverage of $0.71 \mathrm{~m}^{2} / \mathrm{m}^{2}$ by the thin film in the area between the droplets. When the coalescence happens, the droplet motion wipes part of the substrate and leaves a dry area behind. The dry area serves as a new site for drop nucleation. Our high-speed SPRi instrument for thickness measurement provides experimental data that shows-for the first time- the coexistence of a dry area and adsorbed film on the substrate during dropwise condensation. The results of this work cast doubt on film rupture theory as the dropwise condensation mechanism. In future work, we will enhance the sensitivity and spatial resolution of our SPRi instrument to have a more in-depth study on the nucleation phase of dropwise condensation.

Acknowledgements This research was partially sponsored by the National Research Foundation of Korea (NRF) grant that was funded by the Korea government (MSIP) (No. 2017R1A2B2006943, SHL). The authors acknowledge Dr. Fei Long from Michigan Technological University for his assistance on surface roughness characterization by AFM.

Open Access This article is distributed under the terms of the Creative Commons Attribution 4.0 International License (http://creativeco mmons.org/licenses/by/4.0/), which permits unrestricted use, distribution, and reproduction in any medium, provided you give appropriate credit to the original author(s) and the source, provide a link to the Creative Commons license, and indicate if changes were made.

\section{References}

Afkhami B, Wang Y, Miers SA, Naber JD (2019) Experimental determination of flame speed and flame stretch using an optically accessible, spark-ignition engine. Int J Engine Res. https ://doi.org/10.1177/1468087419877718

Ahlers M, Buck-Emden A, Bart H-J (2018) Is dropwise condensation feasible? A review on surface modifications for continuous dropwise condensation and a profitability analysis. J Adv Res 16:1-13

Alabastri A, Tuccio S, Giugni A et al (2013) Molding of plasmonic resonances in metallic nanostructures: dependence of the nonlinear electric permittivity on system size and temperature. Materials 6:4879-4910
Anand S, Son SY (2010) Sub-micrometer dropwise condensation under superheated and rarefied vapor condition. Langmuir 26:17100-17110

Barkay Z (2013) In situ imaging of nano-droplet condensation and coalescence on thin water films. Micros Microanal 20:317-322

Bashkatov AN, Genina EA (2003) Water refractive index in dependence on temperature and wavelength: a simple approximation. In: Proc. SPIE 5068, Saratov fall meeting 2002, optical technologies in biophysics and medicine IV. https://doi.org/10.1117/12.518857

Bayani Ahangar S, Bellur K, Medici E, Tajiri K, Allen JS, Choi CK (2019a) Optical properties and swelling of thin film perfluorinated sulfonic-acid ionomer. ECS Trans 92:197-204

Bayani Ahangar S, Bellur K, Medici E, Tajiri K, Allen JS, Choi CK (2019b) Surface plasmon resonance imaging: an inexpensive tool to study the water transport in thin film PFSA ionomers. ECS Meeting Abstracts MA2019-02:2312

Bayani S, Tabe Y, Kang YT, Lee SH, Choi CK (2018) Surface plasmon resonance imaging of drop coalescence at high-temporal resolution. J Flow Visual Image Process 25:191-205

Beér JM (2007) High efficiency electric power generation: the environmental role. Prog Energy Combust Sci 33:107-134

Beusink JB, Lokate AMC, Besselink GAJ, Pruijn GJM, Schasfoort RBM (2008) Angle-scanning SPR imaging for detection of biomolecular interactions on microarrays. Biosens Bioelectron 23:839-844

Cha H, Xu C, Sotelo J et al (2016) Coalescence-induced nanodroplet jumping. Phys Rev Fluids 1:064102

Cha H, Wu A, Kim M-K, Saigusa K, Liu A, Miljkovic N (2017) Nanoscale-agglomerate-mediated heterogeneous nucleation. Nano Lett 17:7544-7551

Chavan S, Cha H, Orejon D et al (2016) Heat transfer through a condensate droplet on hydrophobic and nanostructured superhydrophobic surfaces. Langmuir 32:7774-7787

Chiang H, Chen C-W, Wu J et al (2007) Effects of temperature on the surface plasmon resonance at a metal-semiconductor interface. Thin Solid Films 515:6953-6961

Cho HJ, Preston DJ, Zhu Y, Wang EN (2017) Nanoengineered materials for liquid-vapour phase-change heat transfer. Nat Rev Mater 2:16092

Choi CK, Margraves CH, English AE, Kihm KD (2008) Multicontrast microscopy technique to dynamically fingerprint live-cell focal contacts during exposure and replacement of a cytotoxic medium. J Biomed Opt 13:054069

Chu F, Wu X, Zhu Y, Yuan Z (2017) Relationship between condensed droplet coalescence and surface wettability. Int J Heat Mass Transf 111:836-841

Daou K, Wang R, Xia Z (2006) Desiccant cooling air conditioning: a review. Renew Sust Energ Rev 10:55-77

de Bruijn HE, Kooyman RPH, Greve J (1993) Surface plasmon resonance microscopy: improvement of the resolution by rotation of the object. App Opt 32:2426

Emonsi H (1939) The mechanism of dropwise condensation. Am Inst Chem Eng Trans 35:109-125

Englert M, Hartmann P, Reichel S (2014) Optical glass: refractive index change with wavelength and temperature. In: Proceedings of SPIE 9131:91310H-91310H-14

Enright R, Miljkovic N, Alvarado JL, Kim K, Rose JW (2014) Dropwise condensation on micro-and nanostructured surfaces. Nanosc Microsc Therm 18:223-250

Hasinoff SW (2014) Photon, poisson noise. In: Ikeuchi K (ed) Computer vision: a reference guide. Springer, Boston, pp 608-610

Homola J (2003) Present and future of surface plasmon resonance biosensors. Anal Bioanal Chem 377:528-539

Homola J (2008) Surface plasmon resonance sensors for detection of chemical and biological species. Chem Rev 108:462-493 
Jakob M (1937) Heat transfer in evaporation and condensation. University of Illinois, Urbana

Jeong CH, Lee SH, Shin DH, Konduru V, Allen JS, Choi CK (2017) High speed SPR visualization of frost propagation inside a subcooled water droplet. J Heat Trans 139:020905

Kandlikar SG, Lu Z (2009) Thermal management issues in a PEMFC stack-a brief review of current status. App Therm Eng 29:1276-1280

Khandekar S, Muralidhar K (2014) Dropwise condensation on inclined textured surfaces. Springer, New York

Kihm KD (2010) Surface plasmon resonance reflectance imaging technique for near-field $(\sim 100 \mathrm{~nm})$ fluidic characterization. Exp Fluids 48:547-564

Kihm KD (2011) Near-field characterization of micro/nano-scaled fluid flows. Springer, New York

Kim IT, Kihm KD (2006) Label-free visualization of microfluidic mixture concentration fields using a surface plasmon resonance (spr) reflectance imaging. Exp Fluids 41:905-916

Kim IT, Kihm KD (2007) Full-field and real-time surface plasmon resonance imaging thermometry. Opt Lett 32:3456-3458

Kim II, Paik S, Jeon YB, Park JS, Kim H, Kim H (2018) Full-field thickness measurement of ultrathin liquid film in receding contact-induced nano-channel using surface plasmon resonance. Opt Express 26:20975-20989

Konduru V, Shin DH, Allen JS et al (2016) High-speed surface plasmon resonance (SPR) reflectance imaging of drop coalescence during condensation and evaporation. J Heat Trans 138:080903

Kretschmann E, Raether H (1968) Radiative decay of non radiative surface plasmons excited by light. Z Naturforsch 23:2135-2136

Langmuir I (1933) Surface chemistry. Chem Rev 13:147-191

Leipertz A (2010) J3 dropwise condensation VDI Heat Atlas. Springer, New York, pp 933-938

Ma J, Cha H, Kim M-K, Cahill DG, Miljkovic N (2019) Condensation induced delamination of nanoscale hydrophobic films. Adv Funct Mater 29(43): 1905222

Miljkovic N, Wang EN (2013) Condensation heat transfer on superhydrophobic surfaces. MRS Bull 38:397-406

Nilsson MA, Rothstein JP (2011) The effect of contact angle hysteresis on droplet coalescence and mixing. J Colloid Interface Sci 363:646-654

Nouri NM, Sekhavat S, Bayani Ahangar S, Faal Nazari N (2012) Effect of curing condition on superhydrophobic surface for $7075 \mathrm{Al}$. J Disper Sci Technol 33:771-774

Oh J, Zhang R, Shetty PP, Krogstad JA, Braun PV, Miljkovic N (2018) Thin film condensation on nanostructured surfaces. Adv Funct Mater 28:1707000

Orejon D, Askounis A, Takata Y, Attinger D (2019) Dropwise condensation on multi-scale bioinspired metallic surfaces with nanofeatures. ACS Appl Mater Interfaces 11(27):24735-24750

Preston DJ, Miljkovic N, Sack J, Enright R, Queeney J, Wang EN (2014) Effect of hydrocarbon adsorption on the wettability of rare earth oxide ceramics. Appl Phys Lett 105:011601

Rahim-Esbo M, Bayani S, Mohammadyari R, Asboei AK, Mohsenian S, Mousavitileboni SE (2014) Analytical and Numerical investigation of natural convection in a heated cylinder using Homotopy Perturbation Method. Acta Scientiarum Technology 36:669-677

Rose JW (2002) Dropwise condensation theory and experiment: a review. P I Mech Eng A J Pow Energy 216:115-128

Rothenhäusler B, Knoll W (1988) Surface-plasmon microscopy. Nature 332:615-617
Ruemmele JA, Golden MS, Gao Y et al (2008) Quantitative surface plasmon resonance imaging: a simple approach to automated angle scanning. Anal Chem 80:4752-4756

Rykaczewski K, Scott JHJ, Rajauria S, Chinn J, Chinn AM, Jones W (2011) Three dimensional aspects of droplet coalescence during dropwise condensation on superhydrophobic surfaces. Soft Matter 7:8749

Rykaczewski K, Anand S, Subramanyam SB, Varanasi KK (2013) Mechanism of frost formation on lubricant-impregnated surfaces. Langmuir 29:5230-5238

Saga K, Hattori T (2005) Organic contamination control in silicon surface processing. Solid State Phenom 103-104:49-54

Schmidt E, Schurig W, Sellschopp W (1930) Condensation of water vapour in film-and drop form. Tech Mech Thermodyn 1:53-63

Sharma CS, Combe J, Giger M, Emmerich T, Poulikakos D (2017) Growth rates and spontaneous navigation of condensate droplets through randomly structured textures. ACS Nano 11:1673-1682

Tammann G, Boehme W (1935) Die zahl der wassertröpfchen bei der kondensation auf verschiedenen festen etoffen. Ann Phys 414:77-80

Tanasawa I (1991) Advances in condensation heat transfer. Advances in heat transfer, vol 21. Elsevier, New York, pp 55-139

Umur A, Griffith P (1963) Mechanism of dropwise condensation. MIT Dept. of Mechanical Engineering, Cambridge

Umur A, Griffith P (1965) Mechanism of dropwise condensation. J Heat Trans 87:275

Wolf LK, Gao Y, Georgiadis RM (2007) Kinetic siscrimination of sequence-specific DNA-drug binding measured by surface plasmon resonance Imaging and comparison to solution-phase measurements. J Am Chem Soc 129:10503-10511

Yamamoto M (2002) Surface plasmon resonance (SPR) theory: tutorial. Rev Polarogr 48:209-237

Yan X, Chen F, Sett S et al (2019a) Hierarchical condensation. ACS Nano 13:8169-8184

Yan X, Huang Z, Sett S et al (2019b) Atmosphere-mediated superhydrophobicity of rationally designed micro/nanostructured surfaces. ACS Nano 13:4160-4173

Yongji S, Dunqi X, Jifang L, Siexong T (1991) A study on the mechanism of dropwise condensation. Int J Heat Mass Transf 34:2827-2831

Zeng Y, Hu R, Wang L et al (2017) Recent advances in surface plasmon resonance imaging: detection speed, sensitivity, and portability. Nanophotonics 6:1017-1030

Zhang L-1, Chen X, Cui D-f (2016a) Performance of surface plasmon resonance imaging system based on angular modulation and intensity measurement. Optoelectron Lett 12:226-228

Zhang X, Tchoukov P, Manica R, Wang L, Liu Q, Xu Z (2016b) Simultaneous measurement of dynamic force and spatial thin film thickness between deformable and solid surfaces by integrated thin liquid film force apparatus. Soft Matter 12:9105-9114

Zhang L, Zhu J, Wilke KL et al (2019) Enhanced environmental scanning electron microscopy using phase reconstruction and Its application in condensation. ACS Nano 13:1953-1960

Zhou C, Jin W, Zhang Y et al (2013) An angle-scanning surface plasmon resonance imaging device for detection of mismatched bases in caspase-3 DNA. Anal Methods 5:2369

Publisher's Note Springer Nature remains neutral with regard to jurisdictional claims in published maps and institutional affiliations. 


\section{Affiliations}

Shahab Bayani Ahangar ${ }^{1} \cdot$ Vinaykumar Konduru ${ }^{1} \cdot$ Jeffrey S. Allen ${ }^{1} \cdot$ Nenad Miljkovic $^{2} \cdot$ Seong Hyuk Lee ${ }^{3}$. Chang Kyoung Choi ${ }^{1}$ (B)

$\triangle$ Seong Hyuk Lee shlee89@cau.ac.kr

$\triangle$ Chang Kyoung Choi cchoi@mtu.edu

1 Mechanical Engineering-Engineering Mechanics, Michigan Technological University, Houghton, MI 49931-1295, USA
2 Mechanical Science and Engineering, University of Illinois at Urbana-Champaign, Urbana-Champaign, IL 61801, USA

3 School of Mechanical Engineering, Chung-Ang University, Seoul 06974, South Korea 